\title{
Les techniques pionnières des premiers spectacles son et lumière
}

Pioneer Techniques of the first Sound and Light Shows

\section{Éric Monin}

\section{(2) OpenEdition}

Journals

Édition électronique

URL : https://journals.openedition.org/rsl/2154

DOI : $10.4000 /$ rsl.2154

ISSN : 2271-6246

Éditeur

Éditions Rue d'Ulm

Référence électronique

Éric Monin, "Les techniques pionnières des premiers spectacles son et lumière », Revue Sciences/ Lettres [En ligne], 6 | 2019, mis en ligne le 10 décembre 2018, consulté le 30 juillet 2021. URL : http:// journals.openedition.org/rsl/2154; DOI : https://doi.org/10.4000/rsl.2154

Ce document a été généré automatiquement le 30 juillet 2021.

(c) Revue Sciences/Lettres 


\section{Les techniques pionnières des premiers spectacles son et lumière}

Pioneer Techniques of the first Sound and Light Shows

\section{Éric Monin}

1 L'architecture n'est pas un spectacle. Inerte, muette, elle provoque pourtant quantité d'émotions qui n'en finissent pas de remuer un public touché au cœur par toute une déclinaison d'effets plastiques plus ou moins singuliers. Par ses formes et les espaces qu'elle organise, par les matériaux, les textures, les transparences et les couleurs qu'elle convoque, par les jeux de lumière fluctuants qui l'investissent, par les rythmes et les échelles qui l'ordonnent, l'architecture sollicite tous les sens comme un feu d'artifice sans cesse rejoué au gré des heures du jour et de la nuit dans un fracas de crissements et de détonations fabuleuses. Mais on n'habite pas un feu d'artifice. Et lorsque le poète souligne les vertus d'un temple d'Hermès comparable «à quelque chant nuptial mêlé de flûtes ${ }^{1}$ ", c'est pour en célébrer « quatre colonnes, un style très simple, [...] l'image mathématique d'une fille de Corinthe ${ }^{2} »$, dont il «reproduit les proportions particulières ${ }^{3} »$. Admirable, l'architecture se laisse contempler par le passant qui "ne voit qu'une élégante chapelle ${ }^{4}$ », un motif d'où s'élève une douce mélodie qui le charme instantanément.

\section{Les prémices d'une idée lumineuse et sonore}

2 Au-delà des pouvoirs rhétoriques d'une architecture "revêtue d'agréments ${ }^{5}$ », il s'agit d'écouter la petite musique qui s'échappe de certains édifices pour entendre les histoires qu'ils ont à nous raconter. Si l'adage veut que les murs aient des oreilles, certains s'évertuent à leur donner une voix capable de libérer des paroles comme dégelées, à l'image de celles du Quart Livre de François Rabelais. En effet, pour André Castelot qui contribua activement au développement de ce nouveau type de divertissement, "c'est Rabelais qui a inventé le spectacle "Son et Lumière"6" où 
l'architecture constitue à la fois le point de départ et le réceptacle de récits déclamés à la belle étoile.

L'histoire de ces spectacles d'un genre nouveau, inventés au début des années 1950, commence à Chambord, un lieu fabuleux qui a profondément marqué l'imaginaire collectif, un château dont la silhouette n'a cessé d'inspirer quantité d'artistes avant d'être exaltée à son tour par les feux des projecteurs couplés à de puissantes émissions sonores.

Illustration 1 - Vue de la grande façade du château de Chambord illuminé pendant le spectacle son et lumière créé en 1952.

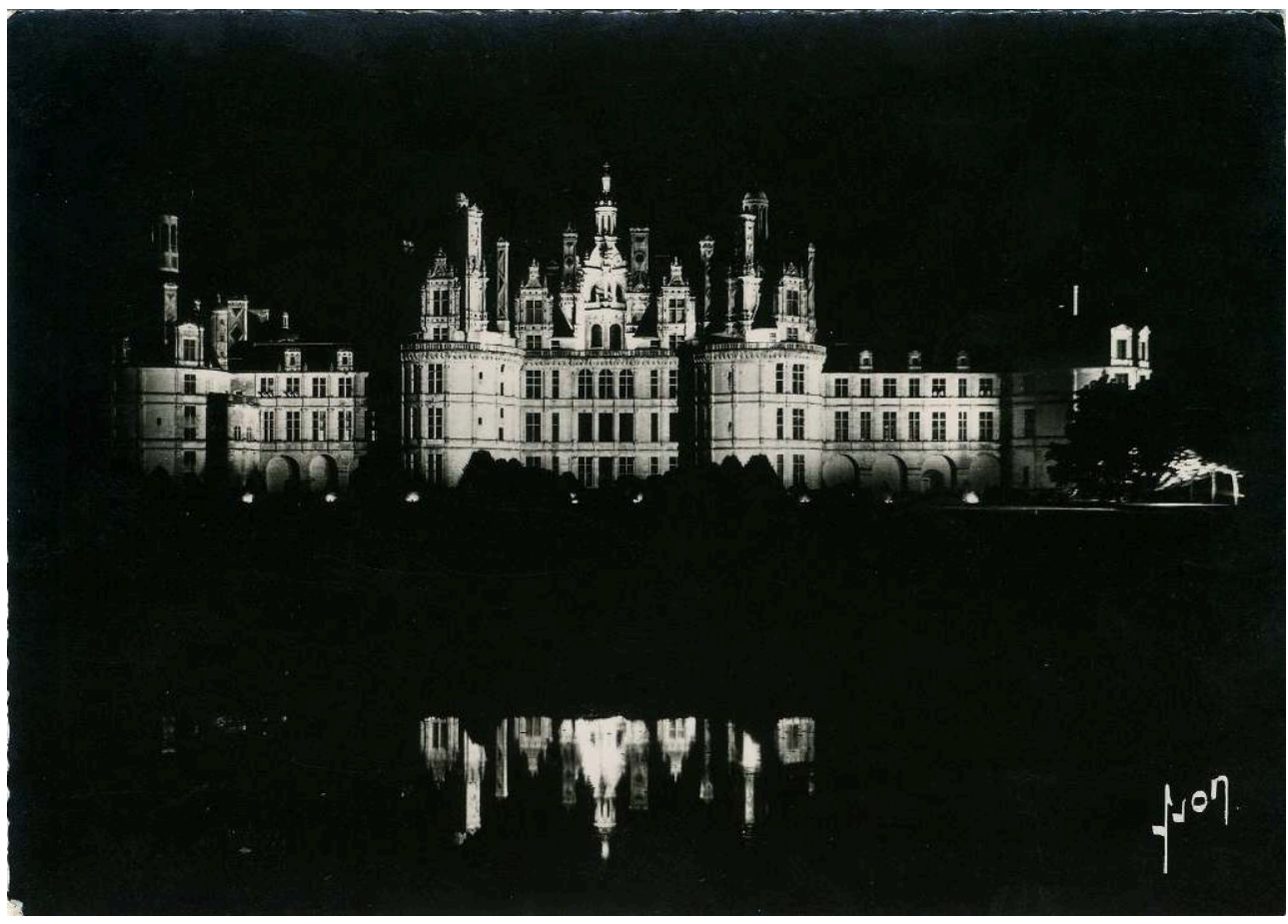

Source : Carte postale, Chambord (L.-et-C.) - Le Château illuminé, Les Éditions d'Art Yvon, Paris.

« De loin l'édifice est une arabesque ${ }^{7}$ » dont le profil résonne déjà comme une mélodie avec ses accords majeurs qui rythment l'ensemble de la composition. Château magique, palais enchanté, le projet tirera parti de nombreuses tentatives de mises en valeur lumineuses réalisées dans l'entre-deux-guerres. En effet, dès les années 1930, une série d'illuminations grandioses avaient déjà été organisées, rassemblant un grand public devant des façades illuminées. Ainsi, à Paris, en 1935 et 1936, le parvis de Notre-Dame accueillait Le Vray Mistère de la Passion d'Arnoul Gréban, spectacle pour lequel le public était rassemblé sur des gradins, face à l'édifice. Chamboulé par cette expérience frontale et grandiose, l'académicien Louis Gillet décrit une «immense et souveraine apparition » où «mille détails inaperçus tressaillaient, soulignés par la puissance des ombres $^{8} »$. Les pouvoirs de l'électricité ne s'arrêtaient pas là et, grâce à elle, les monuments allaient bientôt être sonorisés. Équipés d'« un pick-up et quatre hautparleurs, un jeu de disques divers, choisis pour s'adapter aux différentes illuminations ${ }^{9}$ ", les cinq convois du Tour de France de la Lumière, lancés en 1937 par la Compagnie des Lampes Mazda, célébraient le pouvoir d'une technique capable d'ordonner le spectacle de l'architecture. Mise en lumière et mise en son, comme l'avaient été les spectacles d'eau, de fumigènes et d'artifices conçus pour l'Exposition 
internationale des arts et des techniques organisée la même année à Paris ${ }^{10}$, celle-ci devenait la matière de compositions spectaculaires, polychromes et polyphoniques.

5 Le phénomène prit toute son ampleur quelques années après la Seconde Guerre mondiale quand une heureuse idée permit de réunir le pouvoir de la technique, la force de l'imagination et le pragmatisme de quelques hommes fascinés par le bâtiment et son histoire. Le 21 octobre 1951, après que Paul Robert-Houdin, l'architecte conservateur du château, eut exposé à Pierre Sudreau son souhait de réaliser un «spectacle de Féerie $^{11}$ » autour de l'édifice, le jeune préfet du Loir-et-Cher mit en branle tout son réseau personnel pour donner forme à cette fantasmagorie.

6 Pour exaucer ce doux rêve qui voulait donner la parole au vieux château, la technique fut immédiatement placée au centre d'un projet à peine esquissé mais dont l'échelle constituait la principale gageure. En effet, il fallait inventer les outils permettant de maîtriser une scène de plein air étalée sur 160 mètres de long et 56 mètres de haut. Le cahier des charges indiquait également la nécessité de reproduire l'expérience chaque jour, plusieurs fois par soirée, à heures fixes. D'un point de vue artistique, le château devenait la toile de fond et le personnage principal d'une évocation historique brossée à grands coups de projecteurs et de diffusions sonores. Un peu comme les grottes touristiques réinventées à la lumière des lampes à arcs et des ampoules incandescentes, et dont la visite était parfois rehaussée de diffusions musicales comme au gouffre de l'Aven Armand ${ }^{12}$, Chambord s'apprêtait à revêtir des habits de lumière et de son, pour rejouer son histoire.

\section{La maîtrise totale d'une chaîne sonore}

$7 \mathrm{Au}$ lendemain de la Seconde Guerre mondiale, l'introduction du nouveau projecteur Mazda Infranor P1000 sur le marché de l'éclairage constituait une petite révolution. En permettant d'illuminer intensément de grandes surfaces rectangulaires, son optique était idéalement conçue pour les mises en valeur monumentales. L'adjonction de filtres colorés et l'utilisation de systèmes de commande perfectionnés issus du monde du théâtre facilitaient la production de toute une gamme d'effets chromatiques dynamiques, capable d'animer la grande façade de Chambord.

8 Pour atteindre une qualité d'écoute satisfaisante, la sonorisation du site nécessitait, quant à elle, la résolution d'une série de problèmes autrement plus complexes. Jouer en extérieur n'avait certes rien d'original. Mais ici, "l'acoustique visuelle ${ }^{13}$ ", cette « consonance» entre l'audible et le visible appelée de ses vœux par Le Corbusier, se résumait simplement à « un fronton de pelote basque, sur lequel [venaient] crépiter, comme des grêlons, des molécules de lumière ${ }^{14}$ ", un écran qui avait cependant bien du mal à réfléchir correctement les sons nasillards émis par de modestes haut-parleurs.

En écho aux spectacles de danses serpentines de la Loïe Fuller réalisés quelques décennies plus tôt, et au regard des progrès prodigieux effectués dans le domaine de l'éclairage électrique, Albert de Broglie établissait alors un parallèle direct entre ces deux modes d'expression artistique où la lumière constituait « un Art nouveau fait de mille nuances et de chaudes symphonies ", un art qui est "à l'œil ce que la Musique est à l'oreille ${ }^{15}$ ». Mais l'acoustique bien maîtrisée des music-halls, des théâtres ou des opéras n'a strictement rien à voir avec les propriétés sonores des grands espaces étendus aux pieds des châteaux. Pour donner à ces spectacles monumentaux la chance 
d'être audibles, il fallait réinventer une chaine sonore adaptée, depuis l'enregistrement des voix, jusqu'à l'audition finale.

Bien sûr la puissance du son devait jouer un rôle important dans ces projets pharaoniques, et les racks d'amplificateurs s'entassaient dans les remises et les caves réservées aux postes de commande. Empêchée pendant la guerre de produire ses postes de radio, la société Pathé-Marconi avait profité de cette période de troubles pour développer des compétences pointues dans le domaine des amplificateurs, et c'est elle qui fournirait ce matériel pour Chambord, assorti des transformateurs et des appareils de contrôle nécessaires à la bonne conduite du spectacle. Cependant, comme pour les illuminations transformées par les qualités du projecteur Mazda Infranor P1000, la sonorisation du site bénéficia d'une nouvelle invention qui joua un rôle déterminant dans le succès de ces superproductions. Michel Ranjard, l'architecte en chef des monuments historiques mandaté dès le mois de décembre 1951 par le Secrétaire d'État aux Beaux-Arts pour piloter l'ensemble du projet, souligna très vite la nécessité « de réaliser une sonorisation, non pas seulement statique, mais permettant des effets variés ${ }^{16}$ ", et évoquait pour cela la " possibilité par exemple de donner l'impression que le son parvient de côtés différents ${ }^{17}$ ». Dès ce premier rapport qu'il transmit à sa hiérarchie en janvier 1952, Michel Ranjard soulevait la question essentielle de la mise en mouvement d'un spectacle dépourvu d'acteurs.

Alors que les effets lumineux puisaient leurs sources dans le monde du théâtre, les premiers effets sonores allaient prendre forme à Chambord grâce aux expériences récemment réalisées par les services de la radiodiffusion française. Les choses se sont mises en place un peu par hasard, avec l'activation d'un réseau informel provoquée par Jean Martin-Demézil, l'archiviste en chef du Loir-et-Cher chargé d'écrire le texte du spectacle. Roland-Manuel ${ }^{18}$, qu'il venait de solliciter pour s'occuper de la partition musicale, l'orienta vers le Club d'Essai de la R.T.F. dont quelques membres allaient vite former le noyau dur de nombreux spectacles son et lumière ${ }^{19}$. Essentielle d'un point de vue artistique, la contribution du Club d'Essai fut déterminante d'un point de vue technique, grâce aux travaux de José Bernhart et Jean-Wilfrid Garrett, les co-inventeurs de la stéréophonie dirigée. 
Illustration 2 - Programme de la présentation inaugurale du spectacle Les Très Riches Heures de Chambord, organisée le 30 mai 1952. On mesure bien, dans ce programme, toute la diversité des compétences rassemblées autour du projet.

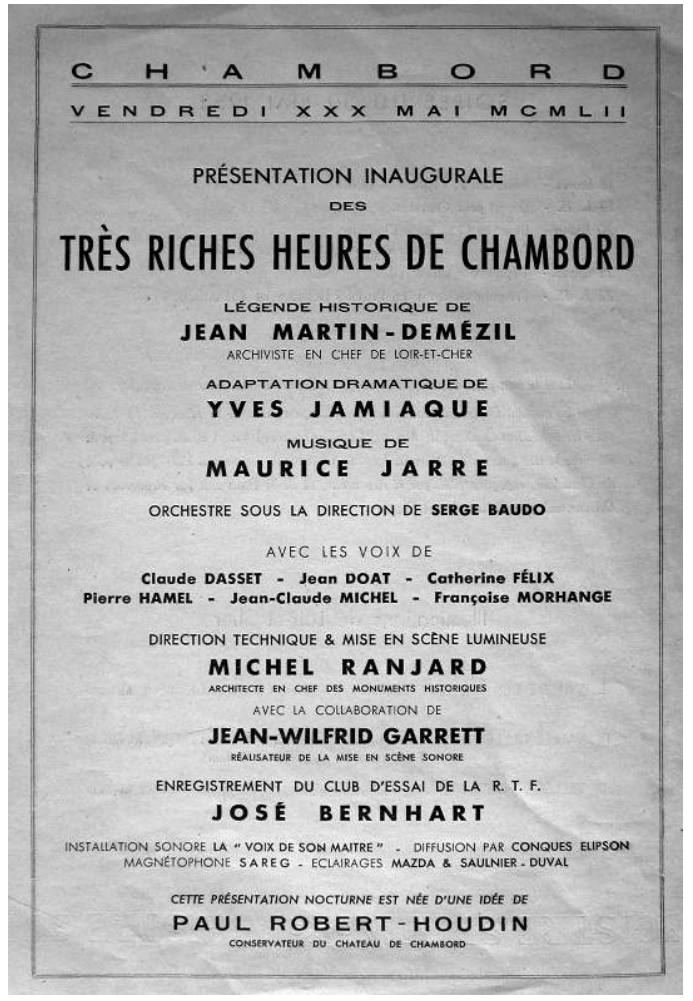

Source : Archives départementales de Loir-et-Cher, 90/J/195

12 Le Traité de prise de son $^{20}$ publié en 1949 par José Bernhart, chef de service à la Radiodiffusion française, expose les grands principes qui serviront à cette invention dont le brevet sera déposé en juillet $1950^{21}$. Dans ce texte qui rassemble des travaux commencés avant-guerre et portant principalement sur les plans sonores, l'auteur se penche sur la question très concrète des problèmes de prise de son à partir d'une étude physique rigoureuse des phénomènes de diffusion et de perception. Cette approche traduit techniquement les préoccupations du Club d'Essai, "laboratoire de l'Art radiophonique ", qui «se devait de produire des œuvres créées pour le micro ${ }^{22}$ ». Dans sa recherche, José Bernhart souligne les lacunes des méthodes de reproduction électroacoustiques usuelles qui ne permettent pas de conserver la sensation directionnelle du son. Déjà pointait l'idée d'organiser le déplacement du son dans l'espace pour produire l'illusion du mouvement, en se libérant du système trop contraignant des têtes artificielles d'enregistrement - des têtes en plâtre avec deux microphones à la place des oreilles - qui imposent ensuite, pour plus d'efficacité, l'utilisation de casques à l'audition ${ }^{23}$. En introduisant les notions de "perspective " et d'" architecture sonore", José Bernhart insiste sur des techniques d'enregistrement qui, en maîtrisant précisément la position des sources par rapport au micro, peuvent créer des «plans d'atmosphères " préalables à une " écoute intelligente » et "dirigé ${ }^{24}$ ». Ces travaux prolongeaient les recherches qu'il avait effectuées en 1942 pendant le stage de Beaune (organisé par Pierre Schaeffer avec la collaboration de Jacques Copeau pour préparer l'ouverture du Studio d'Essai radiophonique), où lui-même avait étudié « les conditions techniques qui permettaient le mieux de traduire l'atmosphère et le climat ${ }^{25}$ ». 
13 La stéréophonie dirigée apportait cependant une autre solution au problème de la spatialisation du son, qui ne s'opérait plus à l'enregistrement, mais à la reproduction. L'originalité du procédé résidait dans le traitement des enregistrements sonores qui, après avoir été effectués séparément et à des niveaux d'intensité différents, étaient montés sur une bande à double piste. Les deux voies autonomes étaient alors transmises simultanément par des procédés classiques, produisant ainsi l'illusion d'une « source virtuelle image » en mouvement. Dans un article paru en 1953 dans la Revue du son ${ }^{26}$, José Bernhart revient sur l'avantage de cette invention qui « réside dans la possibilité de localiser à l'audition l'emplacement des sources sonores et d'opérer une discrimination entre les directions diverses d'où parviennent les sons ${ }^{27}$ ». En privilégiant les différences d'intensité perçues par chaque oreille, plutôt que les différences de phase entre les ondes ou de temps entre les deux fronts d'onde qui permettent, elles aussi, à l'auditeur de se repérer dans l'espace, José Bernhart et JeanWilfrid Garrett ont opté pour la solution la plus simple à réaliser, puisqu'il suffisait de créer électriquement une différence d'intensité entre des enregistrements indépendants effectués à l'aide d'un seul microphone.

14 Couplée aux gradateurs de lumière, la stéréophonie dirigée allait permettre de donner forme au mirage des Très riches heures de Chambord. Mais pour cela, il fallait encore trouver des haut-parleurs à la mesure des effets sonores esquissés. José Bernhart prit alors contact avec Joseph Léon, ingénieur CNAM et directeur de la société Elipson (anciennement Multimoteur S.A.), détentrice d'un brevet d'enceinte acoustique capable de réfléchir et de concentrer les ondes sonores qu'elle émettait en un point situé à l'extérieur du réflecteur. Capables d'apporter « un élément de présence et de naturel à la parole ${ }^{28}$ ", les conques Elipson étaient, à n'en pas douter, parfaitement adaptées à la stéréophonie dirigée. 
Illustration 3 - Publicité pour les conques Elipson parue dans la Revue du Son, $\mathrm{n}^{\circ} 16-17$, juillet-août 1954.

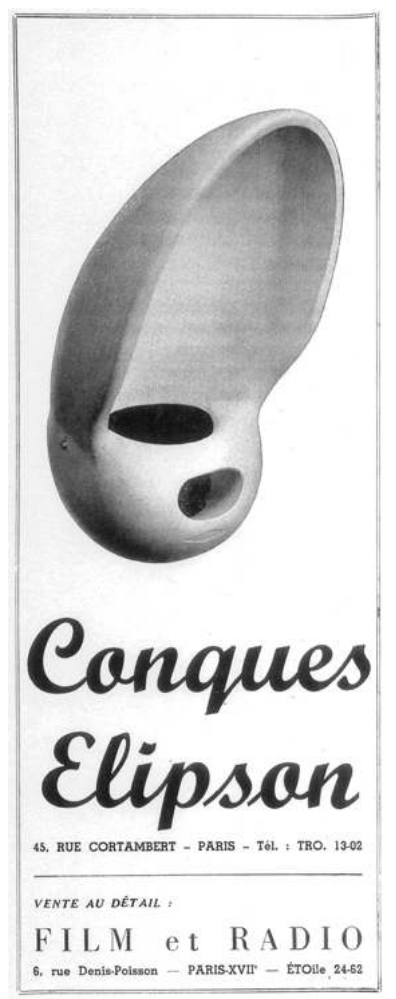

Illustration 4 - Une des conques Elipson utilisées pour le spectacle de Lisieux créé en 1954. On trouve déjà ce dispositif au premier spectacle de Chambord en 1952. Basilique de Lisieux.

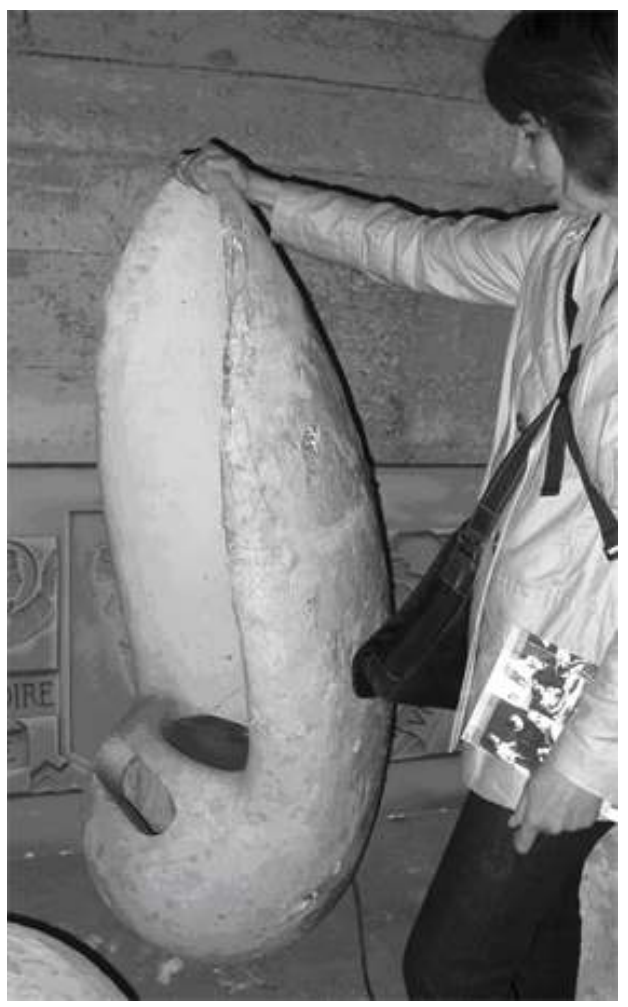

Source : Photo É. Monin 
Déposé le 31 janvier 1950 par la société Multimoteur S.A. ${ }^{29}$, le brevet souligne la forme ellipsoïdale de cet émetteur de son exceptionnel qui se distingue, un peu à la manière du projecteur Mazda Infranor P1000, par ses qualités intrinsèques et par sa forme atypique. Plusieurs décennies après la création de ces diffuseurs, José Bernhart se souvenait encore avec émotion de "ces oreilles d'âne géantes" disposées aux extrémités du château de Chambord et qui laissaient si bien se promener le son devant l'édifice, selon les réglages de balance effectués lors des premiers essais. Ces appareils en plâtre, aux surfaces lisses et aux formes futuristes, ressemblaient à de grands coquillages blancs, des conques dont ils garderont le nom. Comme les projecteurs Infranor, les « conques Elipson » vont elles aussi constituer une marque de fabrique des spectacles son et lumière, en laissant parfois apparaître leur silhouette débordant des toitures des grands monuments illuminés.

Illustration 5 - Photographie des conques Elipson disposées sur la balustrade de la façade du château de Versailles en 1953, extrait de :

"Les fastes de Versailles revivent, grâce à la lumière et au son ", L'Orientation technique, octobre 1953, p. 148.

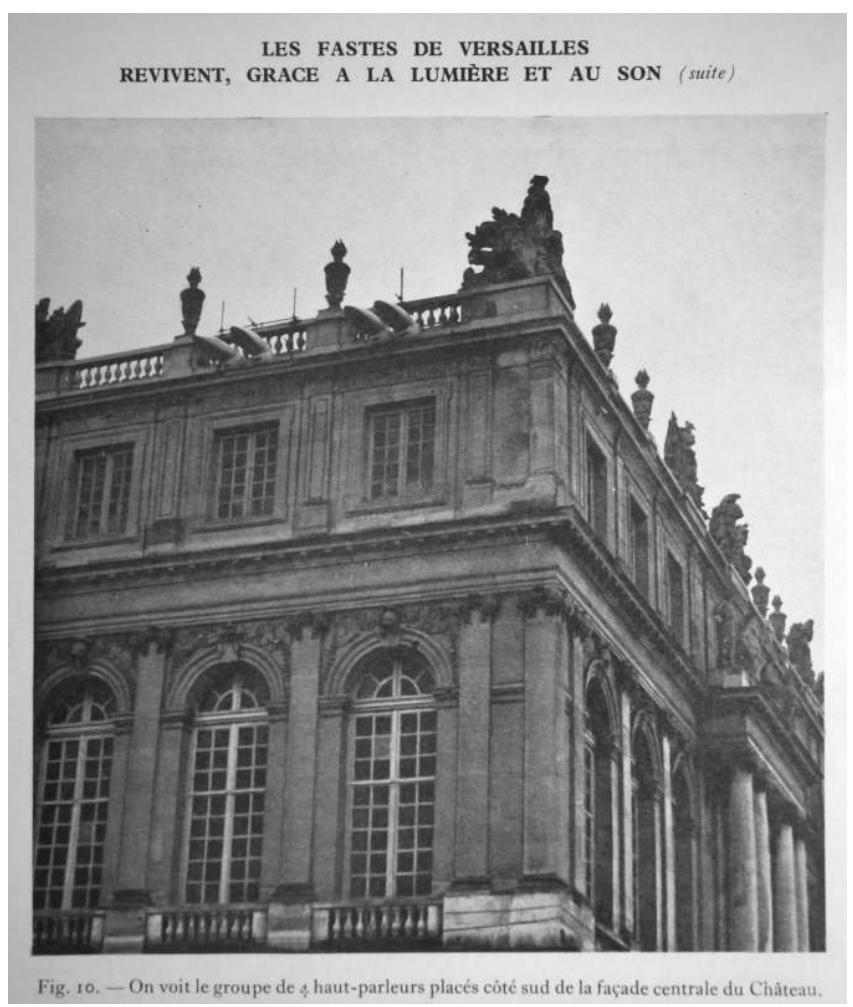

Enfin, contrairement au projet initial qui envisageait d'utiliser de robustes pick-ups pour diffuser des enregistrements réalisés sur disques microsillons, la maison PathéMarconi fut invitée à fournir les magnétophones à plusieurs pistes nécessaires au projet stéréophonique de Chambord. Plus fragiles dans leur utilisation, ces appareils servaient à contrôler l'avancement des spectacles au rythme du défilement des bandes magnétiques et des «TOPS» qui commandaient les éclairages. Tous ces enregistrements et ces réglages étaient réalisés par José Bernhart et Jean-Wilfrid Garrett, à la Radiodiffusion française.

[Lien au dossier pédagogique multimédia en ligne « Entendre le théâtre », à venir début juillet 2019] 
17 Associé au monument, l'ensemble des dispositifs participant à cette chaîne de transmission constituait alors une gigantesque machine à produire des ambiances sonores qui allait connaître une apothéose avec le projet organisé l'année suivante à Versailles : un spectacle dont « la qualité musicale obtenue est, selon Ludovic Gaymard, ingénieur à l'Électricité de France, comparable à une reproduction de très haute fidélité dans une salle ${ }^{30}$ ». Grisé par le succès triomphal du projet, Ludovic Gaymard faisait montre d'un optimisme débordant qui aurait probablement mérité d'être tempéré, même s'il exprimait certainement avec justesse l'impression de perfection que produisait ce spectacle dans le contexte de l'époque. En effet, indépendamment des contraintes physiques des sites investis qui nécessiteront parfois de lourds travaux d'aménagement, couplés aux fréquentes défaillances techniques dues à la fragilité des appareils, les aléas climatiques perturbent régulièrement le bon déroulement des spectacles. Sur le grand plateau du château de Versailles, on observe clairement les effets $\mathrm{du}$ vent qui modifient considérablement la qualité des diffusions sonores, "[altérant] la courbe de réponse, de l'amplitude en fonction de la fréquence », et produisant «un véritable changement des rapports harmoniques des sons, particulièrement sensibles au cours des représentations musicales, les instrumentistes semblant jouer faux par suite de l'effet de distorsion des vagues sonores ${ }^{31}$ ». Parfois, la brume s'invite au spectacle et occasionne, elle aussi, « des affaiblissements de son de plus de $25 \mathrm{~dB}$ par rapport au niveau initial ». Soucieux d'offrir au public de bonnes conditions d'écoute, les organisateurs du spectacle intègrent ces paramètres dans leur projet en faisant "plusieurs enregistrements avec des tonalités très variables et [en équipant] les appareils magnétophones [...] de correcteurs permettant de corriger la courbe de réponse en fonction des données hygrométriques ${ }^{32} »$. Enfin, toujours à Versailles, dans la seconde partie du spectacle, d'autres corrections permettront d'esquiver l'effet de masque des fontaines ${ }^{33}$ pour respecter toutes les nuances des voix d'acteurs.

\section{Un art de l'assemblage}

18 À l'occasion du congrès des conservateurs de musées français réunis à Tours en 1952 , Michel Florisoone, secrétaire général de cette association, soulignait comment le spectacle de Chambord se servait de «l'architecture extérieure du palais, comme d'un clavier sonore, dialogué et lumineux, et comme d'un personnage de théâtre se découpant diversement sur le fond de la nuit ${ }^{34}$ ». Pour lui, «il réussit à conférer au monument une vie imprévue où tout le passé et l'histoire du château se rassemblent dans un rythme de film et de symphonie ". Ce témoignage, qui évoque à la fois la diversité des effets plastiques convoqués pour le spectacle et l'intérêt historique de l'expérience, insiste sur les principales vertus d'un art sonore et lumineux, fait de rigueur et de précision. Comme le cinéma, comme l'interprétation d'un orchestre symphonique, les spectacles son et lumière résultent d'un savant réglage et d'habiles synchronisations permettant à l'ensemble des appareils rassemblés autour de l'édifice de jouer juste.

19 Le succès des spectacles son et lumière relève en effet d'une curieuse alchimie entre l'architecture, l'histoire, les voix, les lumières et l'ensemble des ressources techniques chargées d'agréger tous ces composants de manière harmonieuse sous les yeux du public. Les affiches des spectacles retiennent davantage le nom des auteurs, des 
compositeurs, des réalisateurs et des acteurs qui prêtent leur voix à ces évocations historiques, que celui des techniciens qui préparent les montages finaux dans l'ombre de leurs ateliers. Mais dans ce foisonnement d'essais, d'enregistrements, de coupes et de montages nécessaires à l'établissement de la bande originale, il est pourtant bien difficile de faire la part des choses en distinguant clairement les limites entre l'art et la technique. En effet, au-delà des interprétations captées sur place ou en studio, la mise en scène sonore implique un engagement sensible, où s'exprime un art extrêmement subtil de l'assemblage, du mélange et de la correction, qui permet le déploiement du son dans l'espace investi. En virtuose de la bande magnétique, le metteur en son devient une sorte de peintre d'images sonores, composées à partir d'une riche palette d'enregistrements soigneusement réalisés puis mélangés à dessein pour produire la magie du spectacle. Avec ses machines, il devient le ventriloque qui anime les vieux châteaux célébrés par la puissance des «arts relais ${ }^{35}$ ». Commandées par un enregistrement sonore qui défile à $38 \mathrm{~cm} /$ seconde, les lumières viennent ensuite se caler sur un scénario parfaitement maîtrisé.

Illustration 6 - Conduite lumière du spectacle son et lumière de Chambord. Fiche $n^{\circ} 1$, Introduction du spectacle.

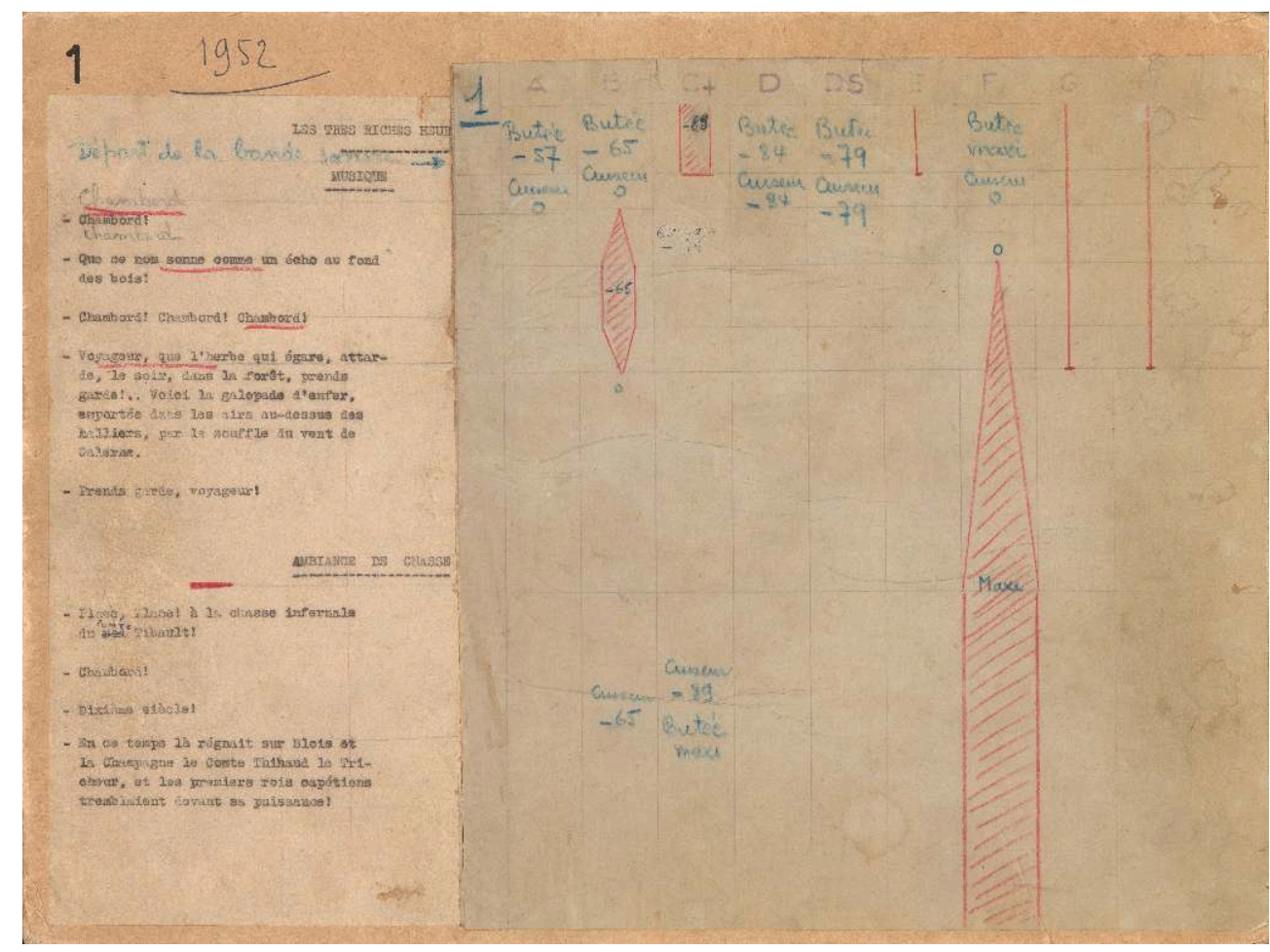

Source : Archives départementales du Loir-et-Cher, Fonds de Chambord 194/4-9.

Comme une pièce radiophonique diffusée en plein air, les récits, la musique, les bruitages donnent au public l'illusion d'une action qui se déroule là, sous ses yeux, rejouée par les fantômes du passé. Diffusés depuis les corniches ou les grandes croisées qui rythment les façades monumentales, les sons ont le pouvoir de raviver l'histoire et de faire voyager les spectateurs dans le temps.

Dès le premier spectacle de Chambord, l'histoire s'est imposée comme une évidence déjà soulignée quelques années plus tôt lors de la première session du Centre d'études philosophiques et techniques du théâtre tenue à la Sorbonne en décembre 1948. 
Pendant cette manifestation consacrée aux «rapports du lieu théâtral avec la dramaturgie présente et à venir", Le Corbusier et Jean Vilar s'accordaient à reconnaître combien la "résonance d'un passé3 ${ }^{36}$ » propre aux lieux investis par le théâtre peut jouer en faveur du spectacle. Ici, c'est le lieu lui-même et son histoire qui dictent le texte d'un spectacle où cohabitent "vérité poétique » et " vérité historique ", " la première tirant de la seconde ses matériaux de construction, opérant un choix, une décantation, mêlant aux voix de l'Histoire celles de la Nature ${ }^{37}$ ». La légende historique imaginée par Jean Martin-Demézil, qui servit de trame directrice pour le spectacle de Chambord, tentait d'opérer cette synthèse à l'attention d'un large public. Et si des résurgences historiques alimentaient l'intrigue du spectacle, Jean Martin-Demézil veillait scrupuleusement à «éviter tout ce qui peut faire cours d'histoire, ou cours d'archéologie ${ }^{38} »$. En effet, pour un chartiste, la tentation est grande, mais celui qui écrira également en 1953 le texte du spectacle Au temps des Dames de Chenonceaux, résistera à l'appel des sirènes académiques en privilégiant une dimension poétique accentuée par les effets sonores et lumineux qui embrassent l'architecture.

Dans cette optique, les textes, les musiques et leurs interprétations sont plus ou moins libérés des faits historiques afin de provoquer une symbiose entre l'architecture et un public qui n'est plus soumis aux compliments récités par des gardiens à casquette. Les accords des musiques de Maurice Jarre, de Jacques Ibert ou de René Cloërec se mêlent aux voix d'Edwige Feuillère, Pierre Fresnay, Jean Topart, Simone Valère, Jean Desailly, Suzanne Flon, Pierre Larquey, Gérard Philipe ou bien encore Maria Casarès pour construire des ambiances propices à l'installation d'une étrange catharsis, sans acteur. Saisi par l'intonation des voix, frappé par les lumières éblouissantes qui tranchent la nuit et alternent avec des fondus colorés, le public entre en communion avec l'architecture qui devient enfin un spectacle. Tirés par les ficelles invisibles de la stéréophonie dirigée, les vieux châteaux se mettent en mouvement comme les créatures d'un marionnettiste capable de faire le jour ou la nuit au gré de ses envies. Paul Robert-Houdin aimait comparer l'expérience à une "séance magistrale d'occultisme ${ }^{39}$ » qui convoque les bruits d'une histoire devenue presque palpable. Les voix qui chuchotent, les ombres qui circulent aux fenêtres de Versailles et donnent aux spectateurs l'impression de voir le Roi Soleil, suscitent avant tout le rêve et l'émerveillement devant des monuments prodigieux qui prennent soudain la parole.

Les avis ne seront pas unanimes, et certains auteurs comme Bernard Rudofsky ou Françoise Choay fustigeront une pratique trop fantaisiste qui abaisse l'architecture au rang de "divertissement mineur ${ }^{40}$ ", investie par des "mécanismes d'ambiance, analogues à ceux que mettent en œuvre les grandes structures commerciales ${ }^{41} »$. Dans un souci de vulgarisation, ces premières réalisations tentaient pourtant d'orchestrer la rencontre de la "grande histoire » avec le patrimoine architectural, au profit de celuici. Jacques Rigaud qui effectuait alors son stage de l'ENA à la préfecture du Loir-et-Cher avait bien compris les enjeux de ce nouveau genre de spectacle capable de mettre la culture à la portée du plus grand nombre. Grâce aux son et lumière, "la visite d'un château est une leçon vivante d'histoire et de culture ", précise-t-il, persuadé qu'il faut " profiter de cette occasion pour réveiller, plus que de vieux souvenirs scolaires, l'esprit d'un peuple ${ }^{42}$ ». Ces spectacles donnaient alors à l'architecture une voix à fleur de pierre, faisant du théâtre de l'histoire le lieu d'expériences scéniques originales qui célébraient une alliance nouvelle entre les arts et les techniques. 


\section{NOTES}

1. Paul Valéry, Eupalinos ou l'architecte, Paris, Librairie Gallimard, 1924, p. 105.

2. Ibid.

3. Ibid., p. 104.

4. Ibid.

5. Charles Batteux, Les Beaux-Arts réduits à un même principe, Paris, Durand, 1747, p. 44.

6. André Castelot, Les Nuits de l'histoire, Paris, Librairie académique Perrin, 1985, p. 7.

7. François René de Chateaubriand, Vie de Rancé, Paris, H. L. Delloye, 1844, p. 74.

8. Louis Gillet, « Jeux de Lumière ", Art et Médecine, mars 1939, [np].

9. «Le "Tour de France de la Lumière" ", Bulletin R.G.E., 10 juillet 1937, t. XLII, n 2, p. 5B-6B. Dans sa livraison du 6 juillet 1937, en prévision des illuminations réalisées à Moulins-sur-Alliers, le journal Le Bourbonnais précise que ce "jeu de disques divers" a été choisi "par un critique musical ». Voir «L'illumination de la Cathédrale et de Jacquemart », Le Bourbonnais, 6 juillet 1937, page de couverture.

10. Conçues par les architectes Eugène Baudouin et Marcel Lods, les Fêtes de la lumière et du son sur la Seine furent jouées à Paris entre le pont de l'Alma et l'île aux Cygnes durant tout l'été 1937. 11. Paul Robert-Houdin, Rapport concernant la présentation nocturne et l'éclairage du Château de Chambord, note tapuscrite, Archives départementales de Loir-et-Cher, SC/135/1.

12. Norbert Casteret, E.-A. Martel, explorateur du monde souterrain, Paris, nrf, Gallimard, 1943, p. 190. Dans cet ouvrage, Norbert Casteret raconte comment dans les années 1930, la salle de la Forêt Vierge de l'Aven Armand fut sonorisée par « de puissants haut-parleurs » qui diffusaient «la Marche des Allobroges ».

13. Le Corbusier, "Le théâtre spontané ", in Architecture et dramaturgie, Centre d'études philosophiques et techniques du théâtre, André Villiers (dir.), Paris, Flammarion, 1950, p. 155.

14. Louis Gillet, op. cit., [n.p.].

15. Albert de Broglie, préface au livre d'André Granet, Décors éphémères, Paris, 1948, p. 7-9.

16. Courrier adressé au Secrétaire général aux Beaux-Arts daté du 15 janvier 1952, p. 6. Médiathèque de l'architecture et du patrimoine, 0081/041/0025.

17. Ibid.

18. Note de Jean Martin-Demézil concernant l'histoire du spectacle de Chambord: « $1^{\circ}$ ) Dans quelles conditions et par l'intermédiaire de qui... ». Archives départementales de Loir-et-Cher, 90/J/195.

19. Jean Martin-Demézil, «Points de vue artistique et touristique des illuminations de monuments ", in Journée de l'éclairage de l'AFE, Monaco (1954), Paris, AFE, 1954. Tiré à part, p. 4.

20. José Bernhart, Traité de prise de son, Paris, Eyrolles, 1949.

21. José Bernhart et Jean-Wilfrid Garrett, Procédé pour la reproduction stéréophonique des sons, France, brevet $\mathrm{n}^{\circ}$ 1021500. 1953-02-19.

22. Éliane Clancier, « Monographie du Club d'Essai de la Radiodiffusion française (1946-1960)», mémoire de maîtrise d'histoire, réalisé sous la direction de Pascal Ory, université Paris 1 Panthéon Sorbonne, septembre 2002, p. 52.

23. Entretien avec José Bernhart réalisé par Bertrand Fillaud le 17 juin 1991 à Paris. Nous tenons à remercier chaleureusement $\mathrm{M}$. Bertrand Fillaud qui a bien voulu mettre cet enregistrement à notre disposition.

24. José Bernhart, op. cit., p. 91-92.

25. Voir note 22. Au cours de ce stage, José Bernhart avait procédé «à des recherches sur l'influence du rapport des densités des sons directs et des sons réfléchis », comme il le rappelle dans l'avant-propos de son traité. 
26. José Bernhart, «Le spectacle de stéréophonie et d'illumination sur la façade du château et dans les jardins du château de Versailles ", Revue du son, n 3, juin 1953, p. 116-119.

27. Ibid., p. 119.

28. Ibid., p. 117.

29. Voir Joseph Léon, Émetteur de sons, Suisse, brevet n²90349.

30. Ludovic Gaymard, "Une réussite de l'esprit d'équipe. La fête de lumière et de son de Versailles », Hommes et techniques, ${ }^{\circ} 110$, février 1954, p. 85.

31. Jean Reyval, "Les illuminations du Château de Versailles", Revue générale de l'électricité, juin 1954, p. 13. Jean Reyval emprunte beaucoup d'éléments à l'excellent article de José Bernhart publié dans la Revue du son: "À propos du spectacle stéréophonique du château de Versailles ", Revue du son, $\mathrm{n}^{\circ}$ 6, septembre 1953, p. 204-206.

32. Ibid., p. 14.

33. À ce sujet, voir Ludovic Gaymard, op. cit., p. 85.

34. Michel Florisoone (secrétaire général), «Compte-rendu du congrès de Tours, 4-7 septembre 1952 », Bulletin trimestriel de l'Association générale des conservateurs des collections publiques de France, $\mathrm{n}^{\circ} 41$, octobre 1952, p. 10.

35. Voir Pierre Schaeffer, "L'art et les machines », Les Cahiers de la recherche : étapes, février 1964, présenté par Sophie Brunet et Carlos Palombini dans Pierre Schaeffer, Essai sur la radio et le cinéma. Esthétique et technique des arts relais 1941-1942, Paris, Éditions Allia, 2010, p. 106.

36. Le Corbusier, art. cit., p. 165.

37. Jean Martin-Demézil, op. cit., p. 5.

38. Ibid., p. 8.

39. C'est ainsi que Paul Robert-Houdin décrit le spectacle qui réveille chaque soir les Dames de Chenonceaux, in La Féerie nocturne des Châteaux de la Loire, Paris, Hachette, 1954, p. 22.

40. Bernard Rudofsky, L'Architecture insolite, Paris, Tallandier, 1979, p. 217.

41. Françoise Choay, L'Allégorie du patrimoine, Paris, Seuil, 1992, p. 167.

42. Jacques Rigaud, L'Action touristique d'un département au Val de Loire: ses fondements, ses buts, ses moyens, mémoire de stage, section économique et financière, École nationale d'administration, promotion Félix Éboué, décembre 1952, Blois, Préfecture de Loir-et-Cher. Archives nationales de Fontainebleau, 20030392 art. 14.

\section{RÉSUMÉS}

Les premiers spectacles son et lumière créés au château de Chambord en 1952 ont permis de réinventer un patrimoine parfois oublié par le grand public. Combinées à de puissants dispositifs de projection lumineuse, les nouvelles techniques de stéréophonie dirigée développées par des ingénieurs du son de la RTF, ainsi que les diffuseurs sonores mis au point par la société Elipson, constituaient les ressources essentielles d'un spectacle où l'architecture était à la fois le fond de scène et le personnage principal. Mise en mouvement par une musique et des textes qui donnaient l'impression de circuler devant les grandes façades monumentales, animée par des effets de lumière ondoyants, l'architecture semblait alors accueillir une histoire qui se rejouait chaque soir d'été, sous les yeux de plusieurs centaines de spectateurs médusés.

L'histoire des premiers spectacles son et lumière raconte des collaborations multiples et 
fructueuses où se sont à la fois manifestés l'esprit d'invention et une formidable envie de redonner leur lustre à des sites prestigieux réinvestis par les ressorts de l'art et de la technique.

The first sound and light shows created at the Château de Chambord in 1952 helped revive a heritage sometimes overlooked by the general public. A combination of powerful light projection devices, new directional stereophonic techniques developed by RTF sound engineers and speakers designed by Elipson constituted the main resources for a show where architecture was both the backdrop and the main character. Music and texts appearing to flow around huge monumental façades were combined with rhythmic lighting effects to convert architecture into the setting for a story enacted each summer evening before hundreds of captivated spectators. The history of the first sound and light shows is characterized by a wealth of productive collaborations demonstrating a spirit of creativity and a dogged determination to restore the original splendour of prestigious sites, which are reinvigorated by the workings of art and technical ingenuity.

INDEX

Mots-clés : stéréophonie dirigée, conques Elipson, spectacles son et lumière, Chambord

Keywords : directional stereophonic sound system, Elipson conch shell speakers, sound and light shows, Chambord

\section{AUTEUR}

\section{ÉRIC MONIN}

Éric Monin est professeur d'histoire et de culture architecturale à l'École nationale supérieure d'architecture et de paysage de Lille.

Parmi ses publications :

«La naissance des premiers spectacles son et lumière : Les Très Riches Heures de Chambord », in

R. Carvais, A. Guillerme, V. Nègre et J. Sakarovitch (dir.), Édifice \& Artifice. Histoires constructives, Paris, Picard, 2010, p. 196-201.

«L'architecture surexposée », in S. Doré, F. Herbin (dir.), L'Architecture exposée, Bourges, ENSA de Bourges, 2015, p. 111-119.

« Et la lumière fut... en architecture », L'Architecture d'aujourd'hui. Perspectives, numéro hors série, Lumières, décembre 2015, p. 8-13.

«Le tourisme des lumières » (avec A. Debal-Morche), in 200 ans de tourisme en Touraine, catalogue de l'exposition éponyme, Tours, Conseil départemental d'Indre-et-Loire, 2016, p. 112-135.

"Festival Architectures as Well-Ordered Entertainment », in H. F. Mallgrave (dir.), The Companions to the History of Architecture, vol. 2, Eighteenth-Century Architecture, Wiley Blackwell Publishers, 2017, p. 285-310.

"Quelle que soit sa fonction, derrière l'outil se cache... ", in R. Klein (dir.), À quoi sert l'histoire de l'architecture aujourd'hui, Paris, Hermann, 2018, p. 127-131.

«La fabrique lumineuse des paysages naturels souterrains », Les Carnets du paysage, $\mathrm{n}^{\circ} 34$, sept. 2018, p. 160-173. 